\title{
Relación entre la riqueza de
}

\section{especies arbóreas y el cambio climático}

\author{
en el sureste de México
}

\section{Relationship between tree species richness and climate change in southeastern Mexico}

\author{
Alberto Santillán-Fernández', Judith Escobar-Castillo², Arely del Rocío Ireta-Paredes³, Ezequiel Espinosa-Grande \\ Jaime Bautista-Ortega ${ }^{4}$ y Bruno Manuel Chávez-Vergara ${ }^{*}$
}

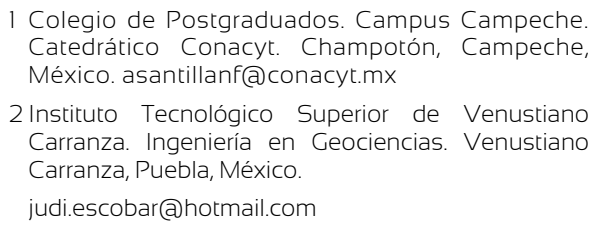

5 Universidad Nacional Autónoma de México. Instituto de Geología. Laboratorio Nacional de Geoquímica y Mineralogía. Ciudad de México, México.

* Autor de correspondencia chavezvb@geologia.unam.mx

\section{RESUMEN}

El sureste de México es la región con la mayor riqueza de especies arbóreas en el país y potencialmente vulnerable a los efectos del clima futuro, por lo cual el objetivo de este trabajo fue relacionar la riqueza de especies y su modificación ante un escenario de cambio climático en el sureste de México. Para este fin se modeló, mediante MaxEnt, la distribución potencial actual y ante el cambio climático de 760 especies arbóreas. Actualmente, el incremento de temperatura puede afectar negativamente la distribución potencial de las especies. Los modelos de cambio climático para 2050 sugieren un incremento en la temperatura, ante lo cual la cobertura arbórea se reducirá; en el caso de las especies que coexisten en selva tropical, se concentrarían en el centro de la región, mientras que, para las especies de bosque templado, se pronostica una reducción mayor a 95\% del área actual. Sin embargo, se observó que las áreas donde coexiste un mayor número de especies tienden a desarrollar una menor contracción de su área de distribución ante las variaciones climáticas futuras.

Palabras Clave: bosque, Campeche, Chiapas, MaxEnt, selva, Yucatán.

\section{ABSTRACT}

The southeast of Mexico is the region with the greatest wealth of tree species in the country and potentially vulnerable to the effects of the future climate, therefore, the objective of this work was to relate the species richness and its modification to a climate change scenario in the southeast of Mexico. To this end, the current potential distribution and climate change of 760 tree species were modeled using MaxEnt. Currently, the temperature negatively increases the potential distribution of the species. Climate change models by 2050 suggest an increase in temperature, whereby tree cover will be reduced; in the case of species that coexist in the rainforest, they would be concentrated in the center of the region, while for temperate forest species, a reduction $>95 \%$ of the current area is forecast. However, it was observed that the areas where a greater number of species coexist tend to develop a smaller contraction of their range in the face of future climatic variations.

KEYWORDS: forest, Campeche, Chiapas, MaxEnt, rainforest, Yucatan. 


\section{INTRODUCCIÓN}

La importancia del estrato arbóreo ha sido documentada desde un enfoque de servicios ecosistémicos, como los bienes y servicios que proporcionan y que contribuyen directamente al bienestar humano, que son de cuatro tipos: provisión, regulación, soporte y cultural (Bennett, Peterson y Gordon, 2009). Los beneficios se observan a escala local, regional y global, como el almacenamiento y captura de carbono, la regulación de la fertilidad del suelo, el control de la erosión, la regulación del balance y el escurrimiento hídrico superficial (Gamfelt et al., 2013). Además, dentro del ecosistema ayudan a mantener la biodiversidad de flora y fauna (Seidl, Rammer y Spies, 2014).

Sin embargo, pese a su importancia, las especies arbóreas también son altamente vulnerables a los efectos del clima y a las actividades antropogénicas como la extracción de madera, sobre todo en los ecosistemas de bosque y selva (Millar, Stephenson y Stephens 2007). Ante este contexto los estudios sobre los efectos del clima futuro en la distribución de las especies arbóreas permiten por un lado desarrollar soluciones integrales para conservar las condiciones actuales de los ecosistemas y por otro aprovecharlas de manera sustentable mediante el manejo forestal (Wardle y Jonsson, 2014).

A escala internacional, existen investigaciones que predicen los efectos del clima futuro en la cobertura y distribución de las especies arbóreas, España (Lloret, 2012), Europa (Lindner et al., 2014), sureste de Asia (Van Zonneveld, Koskela, Vinceti, y Jarvis, 2009a), norte de América (McKenney, Pedlar, Lawrence, Campbell y Hutchinson, 2007) y México (Van Zonneveld, Jarvis, Dvorak, Lema y Leibing, 2009b; Monterroso-Rivas, Gómez-Díaz y Tinoco-Rueda, 2013). En dichas predicciones, el empleo de modelos de distribución potencial y de cambio climático mediante herramientas de sistemas de información geográfica (SIG) es cada vez más frecuente por la simplicidad de su uso y la confiabilidad en sus resultados (Pliscoff y Fuentes-Castillo, 2011).

Los modelos de distribución potencial y de cambio climático para especies arbóreas, obtenidos mediante técnicas de información geográfica, permiten tomar decisiones en cuanto a su conservación o aprovechamiento al determinar las variables climáticas que más inciden en su desarrollo (Phillips, Anderson y Schapire, 2006). Dentro de los modelos de distribución de especies y cambio climático el algoritmo de Máxima Entropía (MaxEnt) tiene las mejores evaluaciones en comparación con otros programas que modelan la probabilidad de ocurrencia y adaptación de especies arbóreas a partir de variables climáticas actuales y futuras (Elith et al., 2011).

MaxEnt ha sido aplicado para predecir los efectos del clima futuro en especies arbóreas como Pinus patula Schiede ex Schltdl. \& Cham. y Pinus tecunumanii F. Schwerdtf. (Van Zonneveld et al., 2009b), Swietenia macrophylla King (GarzaLópez et al., 2016) y Lysiloma latisiliquum (L.) Benth. (GarzaLópez et al., 2018). Sin embargo, los estudios que analizan la riqueza del estrato arbóreo en los ecosistemas ante el cambio climático son escasos (González-Espinosa et al., 2012). En México los estudios sobre los efectos del clima futuro en las coberturas arbóreas son amplios (Gutiérrez y Trejo, 2014), pero en la mayoría de ellos solo se analiza una especie en particular (Garza-López et al., 2018).

\section{OBJETIVOS}

El objetivo general del presente trabajo fue analizar la relación entre la riqueza de especies arbóreas de bosque templado y selva tropical y el cambio climático en el sureste de México; mediante el uso de herramientas de sistemas de información geográfica para conocer la distribución actual de la riqueza arbórea y en un escenario a 2050 y su posible respuesta a las condiciones del clima futuro.

\section{MATERIALES Y MÉTODOS}

\section{Área de estudio}

En este estudio se consideraron los datos georreferenciados de distribución puntual del estrato arbóreo del sureste de México, que incluyeron a los estados de Tabasco, Chiapas, Campeche, Quintana Roo y Yucatán; dados por el inventario nacional forestal y de suelos (Comisión Nacional Forestal [Conafor], 2014). Para diferenciar el estrato arbóreo del 
arbustivo y herbáceo, se discriminaron los registros con individuos de: diámetros menores a $7.5 \mathrm{~cm}$, altura total menor a $1 \mathrm{~m}$ y diámetros de copa menores a $1 \mathrm{~m}$.

\section{Índice de Riqueza de especies arbóreas}

Para ubicar las zonas del sureste mexicano donde coexiste el mayor número de especies arbóreas, se aplicó la técnica Species richness analysis y se estimó el índice de riqueza de especies (IRE), para ello se recurrió al software DivaGis v7.5 (DivaGis, 2019) de sistemas de información geográfica. El tamaño del pixel empleado fue de $5 \mathrm{~km}^{2}$, tamaño recomendado por Warren y Seifert (2011) para estudios de índole regional.

\section{Zonas potenciales de distribución natural}

La distribución puntual in situ de las especies arbóreas obtenida de los datos que se levantaron en campo por Conafor (2014), se relacionaron con variables climáticas y edáficas del sureste de México, con un tamaño de pixel de $5 \mathrm{~km}^{2}$, las primeras corresponden a las 19 variables bioclimáticas y evapotranspiración anual, y las segundas son: calcio, carbono orgánico, potasio, magnesio, materia orgánica, sodio, $\mathrm{pH}$, conductividad eléctrica, y relación de adsorción de sodio; disponibles en la base de datos global de superficies climáticas (WorldClim, 2019).

Los puntos georreferenciados in situ con especies arbóreas se introdujeron en el algoritmo de máxima entropía (MaxEnt, 2019) en formato delimitado por comas (.csv) y las variables climáticas y edáficas en formato ASCII (.asc). Con la metodología de Hijmans, Cameron, Parra, Jones y Jarvis (2005) se determinó la probabilidad de que la especie se desarrolle adecuadamente considerando variables edafoclimáticas. Los resultados se importaron a DivaGis v7.5 (DivaGis, 2019) y se convirtieron en formato grig (.grd) para visualizarse como imágenes.

Para establecer la magnitud en que las variaciones de los factores edafoclimáticos afectan la cobertura potencial de la distribución natural de las especies arbóreas, se recurrió al módulo EcoCrop de DivaGis v7.5 (DivaGis, 2019), en este análisis se consideraron las especies de más amplia cobertura tanto de selva tropical como de bosque de clima templado.

\section{Escenarios de cambio climático}

Para determinar las áreas más vulnerables a las variaciones futuras del clima, de las áreas con cobertura arbórea del sureste mexicano, se aplicó un modelo de cambio climático (MCC) a la distribución puntual, conforme la metodología de Hijmans et al. (2005) y un tamaño de pixel de $5 \mathrm{~km}^{2}$. Para ello, se combinaron los resultados obtenidos mediante el algoritmo de MaxEnt (distribución potencial actual) con proyecciones climáticas a 2050 de modelos de circulación global (promedio CCCMA, HADCM3 y CSIRO) bajo el escenario de emisión A2 que incluyeron 19 variables bioclimáticas (Intergovernmental Panel on Climate Change [IPCC], 2007).

El análisis del MCC permitió generar cuatro escenarios: a) áreas de bajo impacto (1): áreas donde una especie ocurre potencialmente en la actualidad y en el futuro, b) áreas de alto impacto (-1): áreas donde una especie ocurre potencialmente en la actualidad pero que no es apta en el futuro, c) nuevas áreas aptas (2): áreas donde una especie ocurre potencialmente en el futuro las cuales no son aptas para la ocurrencia natural en condiciones actuales, y d) área no apta (0), ni bajo las condiciones actuales ni prevista bajo las condiciones futuras (Hijmans et al., 2005).

\section{Relación de la riqueza de especies arbóreas en la adaptación al cambio climático}

Para probar estadísticamente la relación que existe entre el IRE y los MCC, se contabilizaron la cantidad de pixeles que coincidieron por cada uno de los escenarios climáticos (-1, 0,1 y 2) con los valores obtenidos en el IRE, para ello se empleó la técnica del vecino más próximo de herramientas de SIG; que hizo que todos los pixeles del MCC coincidieran con la categoría más cercana al IRE (González de Andrés, Traba-Díaz, Seoane-Pinilla y Morales-Prieto, 2014). Adicionalmente se estimó un modelo de regresión espacial no lineal considerando a los escenarios del MCC 
como variable dependiente y como independiente a los valores del IRE (DivaGis, 2019).

\section{RESULTADOS Y DISCUSIÓN}

En el sureste de México, la Conafor (2014) registró 65535 sitios de muestreo, en los cuales se encontraron 1578885 árboles, 97.21\% correspondieron a ejemplares de 593 especies diferentes que se desarrollan en selvas tropicales, y el restante $2.79 \%$ a 167 especies diferentes que coexisten en bosques de clima templado (Tabla 1). Campeche (334) y Quintana Roo (270) destacaron como las entidades donde se concentró el mayor número de especies en selva tropical; por el contrario, Chiapas, fue la única entidad de la región de estudio donde se localizaron especies de bosque de clima templado (167).

De las 593 especies que coexisten en selva tropical, ocho de ellas concentraron $32.7 \%$ del total de árboles, destacó Lysiloma latisiliquum con 6.65\% lo que representó más de 100000 ejemplares, distribuidos en su mayoría entre los estados de Campeche y Quintana Roo. En bosque de clima templado, que se localizó en Chiapas, el género Quercus concentró más de 30\% de los árboles (Tabla 2). De acuerdo con Carreón-Santos y Valdez-Hernández (2014) por su naturaleza, la mayor riqueza de especies arbóreas se concentra en selvas, específicamente para México la península de Yucatán es el área con el mayor número (Zamora-Crescencio, García-Gil, Flores-Guido y Ortiz, 2008). Sin embargo, el bosque de clima templado alberga las especies de mayor valor comercial, lo que hace a este ecosistema más vulnerable a cambios en la distribución natural de las especies como consecuencia del manejo forestal para la extracción de madera (Granados-Sánchez, López-Ríos y Hernández-García, 2007).

\section{Riqueza de especies arbóreas}

$\mathrm{Al}$ aplicar la técnica de Species richness analysis (riqueza de especies) a los datos georreferenciados con distribución puntual de especies arbóreas, se ubicaron espacialmente las regiones en el sureste de México donde coexisten el mayor número de especies (Fig. 1). Los resultados indican que entre los límites estatales de Campeche y Quintana Roo se encuentraron las zonas con mayor riqueza de especies. Estas zonas se caracterizan por tener precipitaciones entre $450 \mathrm{~mm}$ y $1500 \mathrm{~mm}$, temperatura media anual de $26^{\circ} \mathrm{C}$ y altitud de $0 \mathrm{~m}$ a $400 \mathrm{~m}$ s.n.m., condiciones óptimas para el desarrollo de selva alta y baja perennifolia (Instituto Nacional de Estadística y Geografía [Inegi], 2016).

TABLA 1. Número de especies, sitios de muestreo y número de árboles en el sureste de México conforme los datos del Inventario Nacional Forestal y de Suelos 2014.

\begin{tabular}{lccccccc}
\hline Estado & \multicolumn{3}{c}{ Selva tropical } & & \multicolumn{3}{c}{ Bosque templado } \\
\cline { 2 - 3 } \cline { 6 - 8 } & Especies & Sitios & Árboles & & Especies & Sitios & Árboles \\
\hline Campeche & 334 & 38545 & 962288 & & 0 & 0 & 0 \\
Chiapas & 208 & 2286 & 38351 & & 167 & 2702 & 43994 \\
Quintana Roo & 270 & 15742 & 377595 & & 0 & 0 & 0 \\
Tabasco & 42 & 159 & 2210 & & 0 & 0 & 0 \\
Yucatán & 194 & 6101 & 154447 & & 0 & 0 & 0 \\
Total & 593 & 62833 & 1534891 & & 167 & 2702 & 43994 \\
\hline
\end{tabular}


TABLA 2. Especies con mayor presencia de árboles en selva tropical y bosque templado del sureste de México conforme los datos del Inventario Nacional Forestal y de Suelos 2014.

\begin{tabular}{|c|c|c|c|}
\hline \multicolumn{2}{|c|}{ Selva tropical } & \multicolumn{2}{|c|}{ Bosque templado } \\
\hline Especies & Cobertura (\%) & Especies & Cobertura (\%) \\
\hline Lysiloma latisiliquum (L.) Benth. & 6.65 & $\begin{array}{l}\text { Quercus germana Schltdl. \& } \\
\text { Cham. }\end{array}$ & 14.45 \\
\hline Bursera simaruba (L.) Sarg. & 5.18 & Quercus rugose Neé. & 10.51 \\
\hline Lonchocarpus xuul Lundell & 4.91 & $\begin{array}{l}\text { Pinus oocarpa Schiede ex } \\
\text { Schltdl }\end{array}$ & 3.1 \\
\hline Piscidia piscipula (L.) Sarg. & 3.85 & Quercus sapotifolia Liebm. & 2.87 \\
\hline Gymnopodium floribundum Rolfe. & 3.49 & Quercus skinneri Benth. & 1.78 \\
\hline Croton reflexifolius Kunth. & 2.98 & Quercus conspersa Benth. & 1.6 \\
\hline Thouinia paucidentata Radlk. & 2.9 & Quercus crassifolia Bonpl. & 1.43 \\
\hline Manilkara zapota (L.) P. Royen. & 2.74 & Quercus benthamii A. DC. & 1.37 \\
\hline Otras (585) & 67.3 & Otras (159) & 62.89 \\
\hline Total (593) & 100 & Total (167) & 100 \\
\hline
\end{tabular}

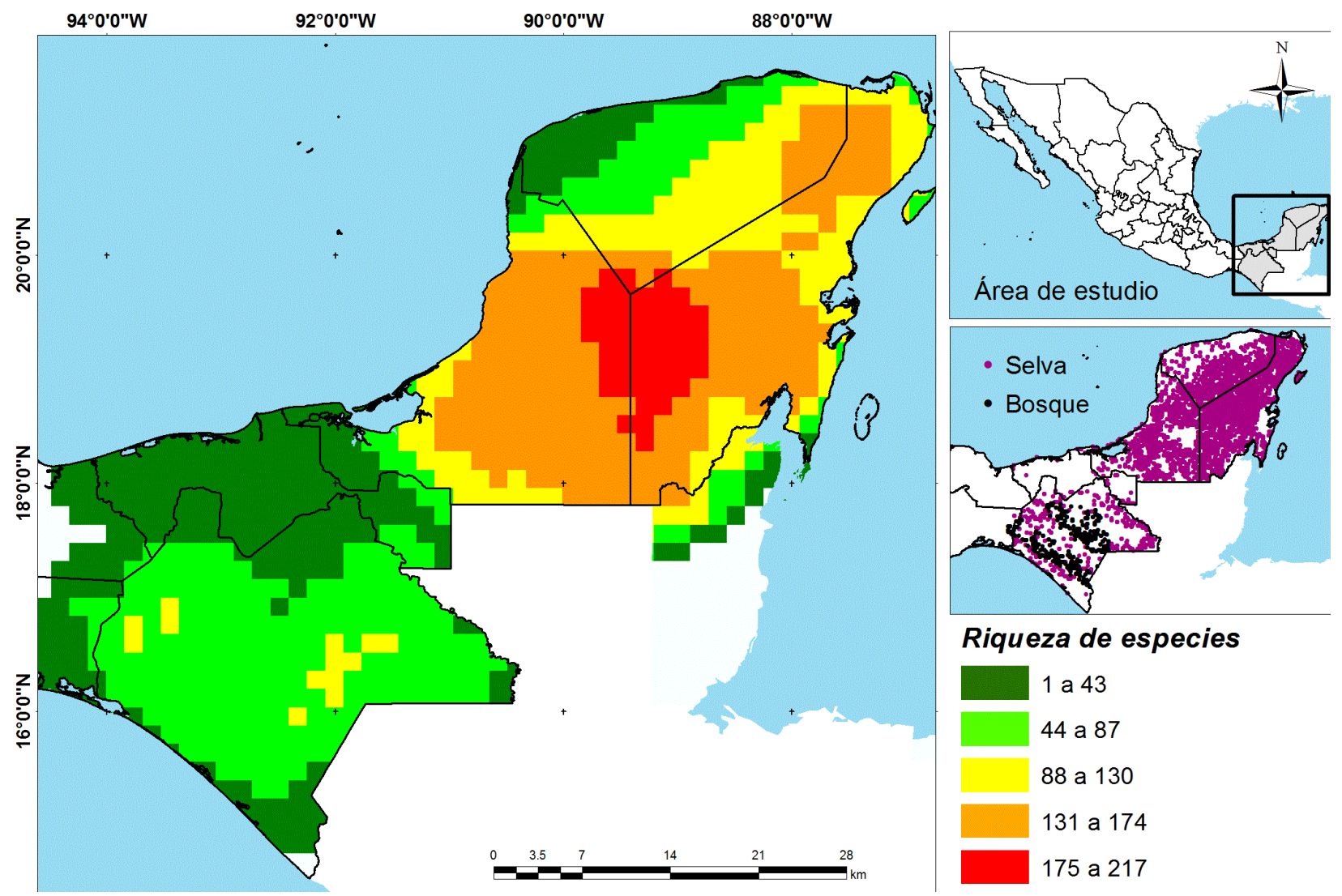

FigurA 1. Distribución espacial de las regiones en el sureste de México con mayor presencia de especies arbóreas, obtenidas mediante la técnica Species richness analysis de Sistemas de Información Geográfica. 


\section{Zonas potenciales de distribución natural}

La distribución potencial natural actual se basó en el registro puntual de 65535 sitios de muestreo, donde se localizaron 1578885 árboles de 760 especies diferentes. Se calculó tanto para especies de selva tropical (593 en 62833 sitios de muestreo) como de bosque de clima templado (167 en 2702 sitios). De acuerdo con Baldwin (2009) se considera un tamaño de muestra suficiente que garantiza predicciones confiables de MaxEnt.

Los resultados obtenidos después de 500 iteraciones fueron altamente significativos con un nivel de confiabilidad de 95\% (P-Value < 0.0001) y un coeficiente de ajuste (AUC = Área bajo la curva) de 98.4\% para bosque templado y $89.1 \%$ para selva tropical. En la tabla 3 (selva) y tabla 4 (bosque) se muestra el efecto que las principales variables edafoclimáticas presentaron en la distribución potencial natural de las especies arbóreas del sureste de México. Los gradientes de temperatura resultaron ser las variables para ambos ecosistemas que más condicionaron su distribución natural. Este aspecto ha sido documentado por Gómez-Mendoza, Galicia y Aguilar-Santelises (2008) y Ramirez-Villegas, Jarvis y Läderach (2013) quienes coinciden que en el sureste de México el incremento de la temperatura reducirá la cobertura de las especies, con un efecto menor en regiones con mayor riqueza de especies.

\section{Escenarios de cambio climático}

El hábitat climático futuro (2050) para las especies arbóreas en el sureste de México que se proyectó mediante el algoritmo de MaxEnt, resultó ser significativo con un coeficiente de ajuste (AUC) del 0.890 obtenido después de 500 iteraciones. El AUC es una medida directa de la capacidad de discriminación del modelo, que toma valores próximos a 1 cuando existe un buen ajuste con los datos de evaluación y cercanos a 0.5 cuando el ajuste no es mejor que el obtenido por azar (Fielding y Bell, 2007).

TABLA 3. Variables edafoclimáticas y su relación con la distribución natural actual de las especies arbóreas de selva tropical en el sureste de México.

\begin{tabular}{|c|c|c|c|}
\hline Variable & $\begin{array}{l}\text { Valor de } \\
\text { referencia }\end{array}$ & $\begin{array}{c}\% \\
\text { Contribución }\end{array}$ & $\begin{array}{l}\text { Efecto en la Cobertura } \\
\qquad(\mathrm{C})^{*}\end{array}$ \\
\hline S1: Temperatura mínima del mes más frio (diciembre) & $16^{\circ} \mathrm{C}$ & 36.2 & $\begin{array}{l}\text { S1: Aumenta; C: } \\
\text { Disminuye }\end{array}$ \\
\hline S2: Rango anual de temperatura & 16 a $36^{\circ} \mathrm{C}$ & 23.3 & $\begin{array}{l}\text { S2: Aumenta; C: } \\
\text { Disminuye }\end{array}$ \\
\hline S3: Materia orgánica & $1 \%$ a $2 \%$ & 9.4 & $\begin{array}{l}\text { S3: Aumenta; C: } \\
\text { Aumenta }\end{array}$ \\
\hline S4: Temperatura promedio anual & $26^{\circ} \mathrm{C}$ & 10.8 & $\begin{array}{l}\text { S4: Aumenta; C: } \\
\text { Disminuye }\end{array}$ \\
\hline S5: Precipitación promedio anual & $1207 \mathrm{~mm}$ & 4.1 & $\begin{array}{l}\text { S5: Aumenta; C: } \\
\quad \text { Aumenta }\end{array}$ \\
\hline $\begin{array}{l}\text { S6: Temperatura promedio en el trimestre más frio (diciembre- } \\
\text { enero-febrero) }\end{array}$ & $<22^{\circ} \mathrm{C}$ & 3.5 & $\begin{array}{l}\text { S6: Aumenta; C: } \\
\text { Disminuye }\end{array}$ \\
\hline Otras (23) & & 12.7 & \\
\hline Total (29) & & 100 & \\
\hline
\end{tabular}

*Estimación desarrollada en EcoCrop (DivaCis, 2019) considerando las especies de más amplia cobertura 
TABLA 4. Variables edafoclimáticas y su relación con la distribución natural actual de las especies arbóreas de bosque de clima templado en el sureste de México.

\begin{tabular}{|c|c|c|c|}
\hline Variable & $\begin{array}{l}\text { Valor de } \\
\text { referencia }\end{array}$ & \% Contribución & $\begin{array}{l}\text { Efecto en la Cobertura } \\
(\mathrm{C})^{*}\end{array}$ \\
\hline B1: Temperatura promedio anual & $24{ }^{\circ} \mathrm{C}$ & 50.4 & $\begin{array}{l}\text { B1: Aumenta; C: } \\
\text { Disminuye }\end{array}$ \\
\hline B2: Materia orgánica & $2 \%$ a $3.5 \%$ & 20.4 & $\begin{array}{l}\text { B2: Aumenta; C: } \\
\text { Aumenta }\end{array}$ \\
\hline B3: Rango anual de temperatura & $17{ }^{\circ} \mathrm{C}$ a $30^{\circ} \mathrm{C}$ & 10 & $\begin{array}{l}\text { B3: Aumenta; C: } \\
\text { Disminuye }\end{array}$ \\
\hline $\begin{array}{l}\text { B4: Temperatura promedio del trimestre más caluroso (abril- } \\
\text { mayo-junio) }\end{array}$ & $23{ }^{\circ} \mathrm{C}$ & 6.4 & $\begin{array}{l}\text { B4: Aumenta; C: } \\
\text { Disminuye }\end{array}$ \\
\hline $\begin{array}{l}\text { B5: Temperatura promedio del trimestre más seco } \\
\text { (diciembre-enero-febrero) }\end{array}$ & $19^{\circ} \mathrm{C}$ & 3.9 & $\begin{array}{l}\text { B5: Aumenta; C: } \\
\text { Disminuye }\end{array}$ \\
\hline Otras (24) & & 8.9 & \\
\hline Total (29) & & 100 & \\
\hline
\end{tabular}

*Estimación desarrollada en EcoCrop (DivaGis, 2019) considerando las especies de más amplia cobertura

De las 19 variables bioclimáticas empleadas para predecir el hábitat climático futuro de las especies arbóreas en el sureste de México, tres contribuyeron con $89.3 \%$ de la predicción, este modelo no considera variables edáficas. El incremento en el rango anual de temperatura (BIO7) aportó $56.1 \%$, que se relaciona con el incremento en la temperatura media anual (BIO1) que contribuyó con $28.2 \%$ y los descensos en la precipitación promedio anual (BIO12) con $5.0 \%$. De acuerdo con el modelo de zonas potenciales actuales, existe una relación inversa entre la temperatura y la cobertura arbórea tanto para las especies que se desarrollan en bosque de clima templado como para las de selva tropical, lo que implica que a medida que las temperaturas se incrementen, las coberturas arbóreas en el sureste de México tenderán a disminuir.

Estos resultados coinciden con los de Orellana, Espadas, Conde y Gay (2012) quienes pronostican para 2020 un incremento en las temperaturas y reducción en las precipitaciones para la península de Yucatán, lo que provocará sequias prolongadas en las costas de la misma, afectando la distribución natural de las especies arbóreas. Al respecto Garza-López et al. (2016 y 2018) estiman para las especies forestales Swietenia macrophylla y Lysiloma latisiliquum reducciones en sus coberturas actuales en el sureste de México de 60\% y 43\% respectivamente como consecuencia de las variaciones del clima futuro para 2050.

En la figura 2 se presenta de manera espacial la proyección para 2050 del hábitat climático futuro de las especies arbóreas del sureste mexicano y su relación con las zonas potenciales actuales y la riqueza de especies. Se observa que la cobertura arbórea de mayor vulnerabilidad (áreas de alto impacto: áreas donde una especie ocurre potencialmente en la actualidad pero que no es apta en el futuro) son aquellas donde la riqueza de especies es menor; de acuerdo con Lloret (2012) las regiones donde coexisten un mayor número de especies tienden a desarrollar mayor adaptación a las variaciones climáticas. 


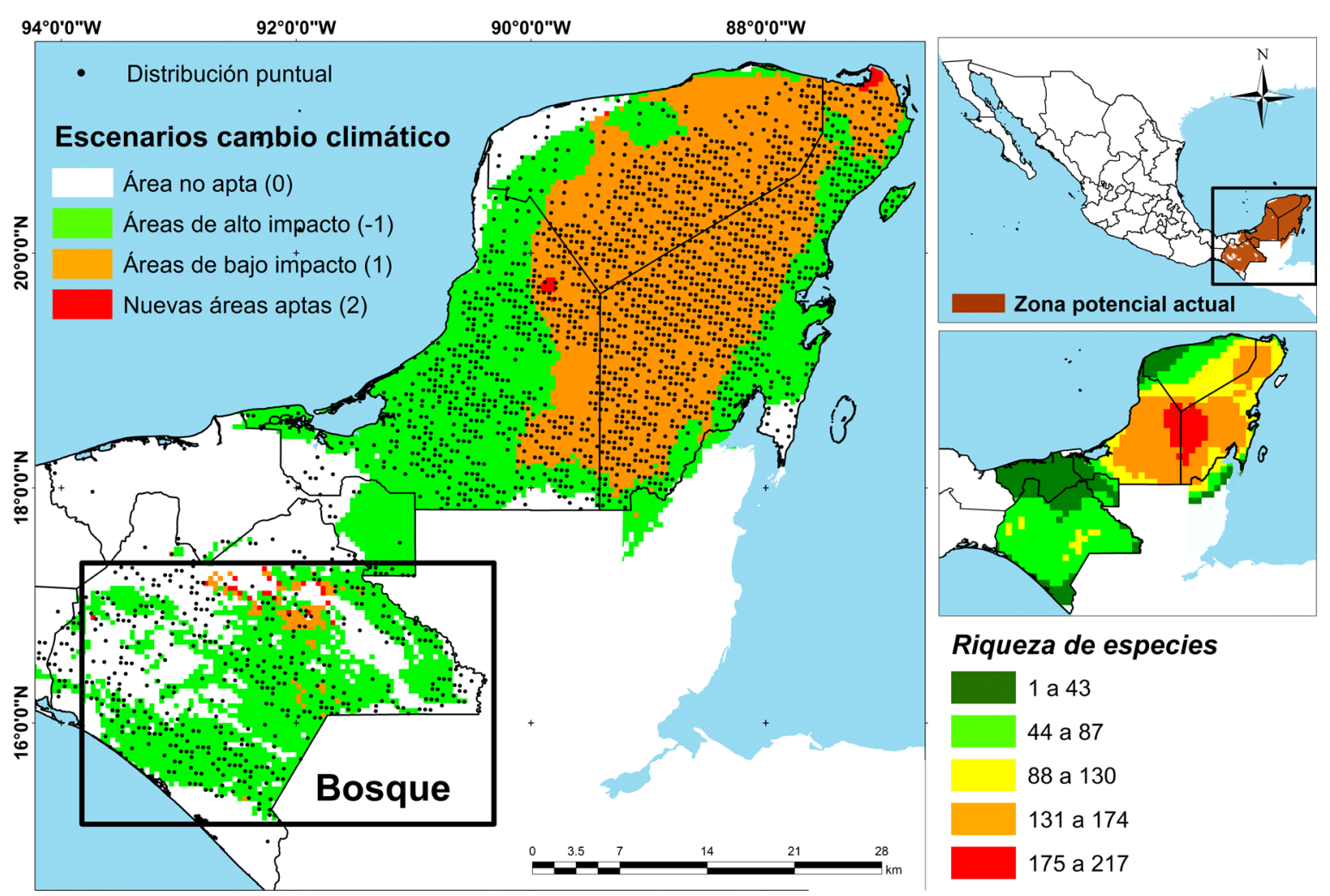

FIGURA 2. Representación espacial del hábitat climático futuro para las especies arbóreas en el sureste de México, obtenida a partir de modelos de cambio climático en MaxEnt para 2050.

El área donde se desarrollan las especies arbóreas en el sureste de México, de acuerdo con el modelo de cambio climático, se verá reducida, se espera que para el caso de las especies que se desarrollan en selva tropical, sus coberturas se alejen de las costas y se concentren en el centro de la península de Yucatán; para el caso de las especies de bosque de clima templado, los escenarios son más drásticos, al pronosticarse una reducción de más de 95\%. Al respecto Matías (2012) argumenta que las especies arbóreas de bosque son más vulnerables a los efectos del cambio climático por la dominancia de una sola especie en superficies más amplias, a diferencia de las zonas de selva donde existe mayor diversidad.

Gutiérrez y Trejo (2014) pronostican para 2050 que en México el cambio climático reducirá en 69\% la distribución potencial del género Quercus, estiman que el sureste del país será la región más afectada. Aunado a ello Díaz, Correa y Méndez (2020) encontraron que las especies de bosque templado son las más usadas como fuente de energía primaria (leña) en las comunidades rurales de México, lo que contribuye a su deforestación. En el sureste de México se concentra $60 \%$ de la población rural del país (Inegi, 2014).

\section{Relación de la riqueza de especies arbóreas en la adaptación al cambio climático}

En la figura 3 se observa que más de $50 \%$ de los pixeles del MCC se concentraron en las categorías más bajas del IRE que a su vez se asocian con los escenarios de efectos de cambio climático alto (-1: áreas de alto impacto). Estos resultados coinciden con lo observado por Gutiérrez y Trejo (2014) quienes encontraron que, para especies arbóreas de bosque templado en México, a medida que el número de especies aumenta, los efectos del cambio climático son menores; esto se explica según Reece y Noss (2014) por la diversidad ecológica. 


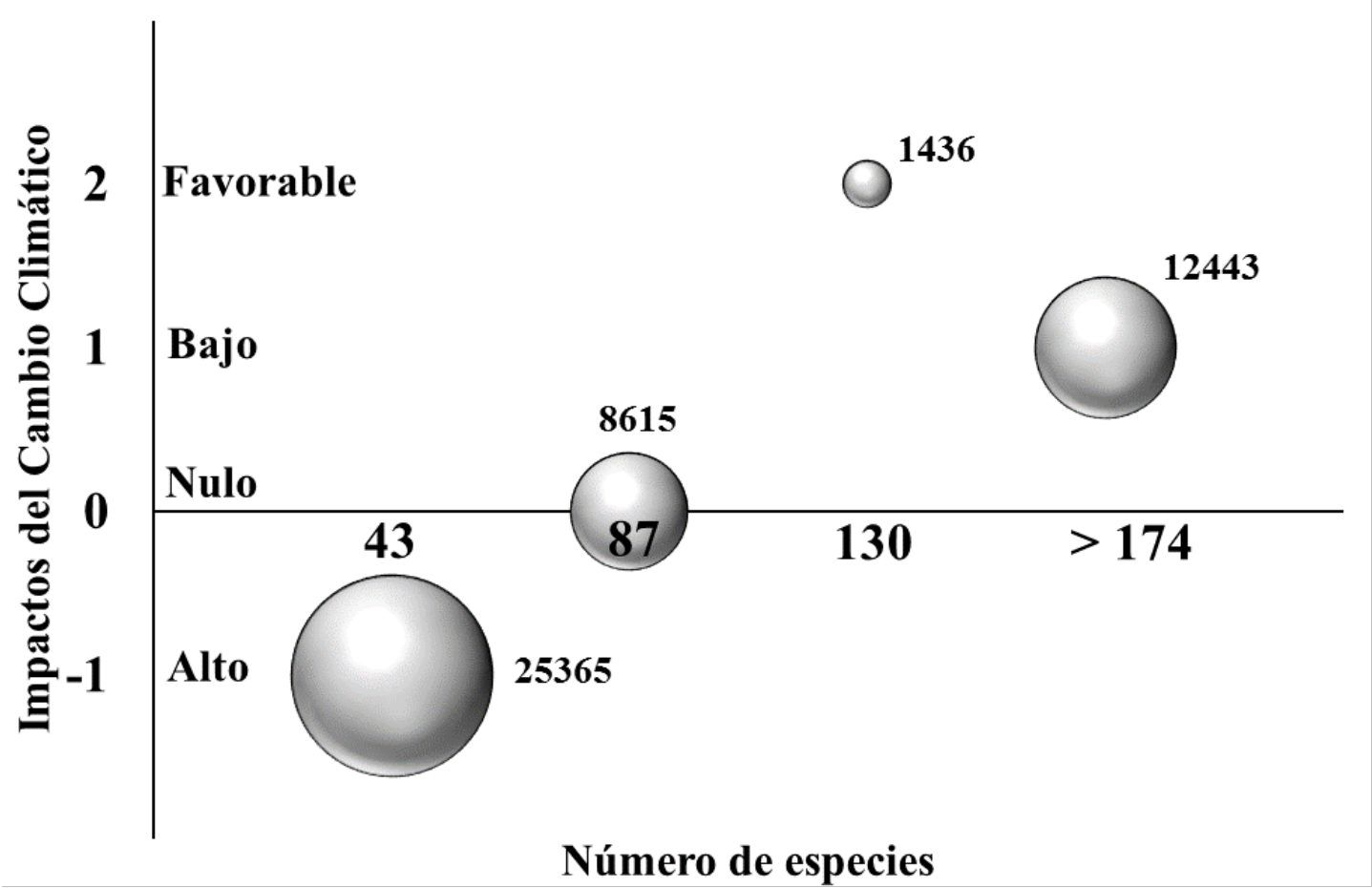

FIGURA 3. Relación de número de pixeles que coinciden para las categorías del modelo de cambio climático (-1, 0 , 1 y 2) con los valores del índice de riqueza de especies obtenida mediante el procedimiento del vecino más cercano de sistemas de información geográfica.

Para comprobar estadísticamente la bondad del ajuste anterior, se desarrolló un modelo de regresión espacial no lineal en DivaGis v7.5 (DivaGis, 2019) mediante la instrucción analysis/regression, donde se consideró como variable dependiente al MCC y como independiente al IRE. El modelo ajustado MCC $=0.0586+0.0875 * \ln (\mathrm{IRE})$ resultó poco significativo con un coeficiente de determinación $\left(\mathrm{R}^{2}\right)$ de 0.0096 . Sin embargo, de acuerdo con Elith et al. (2011) en la predicción de MCC lo que interesa, es el signo de la pendiente de regresión ya que determina la relación que existe entre las variables analizadas. De esta forma el modelo de regresión espacial ajustado confirmó que, a mayor riqueza de especies existente en una región, los efectos del cambio climático serán menores.

\section{CONCLUSIONES}

Se espera que la cobertura espacial con especies arbóreas en el sureste de México se vea reducida con base en el escenario climático a 2050; para el caso de las especies que su hábitat está en el bioma de selva tropical, sus coberturas se alejarían de las costas y se concentrarían en el centro de la región; para el caso de las especies de bosque templado, se pronostica una reducción de más de 95\% del área actual. Sin embargo, un aspecto a resaltar de la investigación es que se observó que las áreas donde coexisten un mayor número de especies arbóreas tienden a desarrollar una mayor capacidad de mantener su distribución ante las variaciones climáticas futuras. Por tanto, en las estrategias de conservación o manejo se debe considerar a la riqueza de especies como un agente del ecosistema que amortigua los efectos negativos del clima futuro.

\section{RECONOCIMIENTOS}

Este trabajo forma parte del proyecto número 364. Reconversión productiva sustentable para el desarrollo de los productores rurales de Campeche, asignado al primer 
autor por parte del Consejo Nacional de Ciencia y Tecnología (Conacyt). A los revisores anónimos del escrito, por sus comentarios, que ayudaron a enriquecer la investigación.

\section{REFERENCIAS}

Baldwin, R.A. (2009). Use of maximum entropy modeling in wildlife research. Entropy, 11(4), 854-866. doi: 10.3390/e11040854

Bennett, E. M., Peterson, G. D., \& Gordon, L. J. (2009). Understanding relationships among multiple ecosystem services. Ecology Letters, 12(12), 1-11. doi: 10.1111/j.1461-0248.2009.01387.x

Carreón-Santos, R. J. \& Valdez-Hernández, J. I. (2014). Estructura y diversidad arbórea de vegetación secundaria derivada de una selva mediana subperennifolia en Quintana Roo. Revista Chapingo Serie Ciencias Forestales y del Ambiente, 20(1), 119-130. doi: 10.5154/r.rchscfa.2013.06.023

Comisión Nacional Forestal [Conafor] (2014). Inventario Nacional Forestal $y$ de Suelos. Recuperado de http://transparencia01.cnf.gob.mx/OpenData/Inventario/INF YS_Microcuenca_2009_2014/

Díaz, M. G., Correa, A. R., \& Méndez, R. M. (2020). Especies vegetales con uso combustible por comunidades rurales mexicanas. Revista Etnobiología, 18(3), 113-135.

DivaGis (2019). A geographic information system for the analysis of species distribution data (Version 7.5). Recuperado de http://www.divagis.org/download

Elith, J., Phillips, S., Hastie, T., Dudík, M., Chee, Y., \& Yates, C. (2011). A statistical explanation of MaxEnt for ecologists. Diversity and Distributions, 17(1), 43-57. doi: 10.1111/j.1472-4642.2010.00725.x

Fielding, A. H., \& Bell, J. (2007). A review of methods for the assessment of prediction errors in conservation presence absence models. Environmental Conservation, 24(1), 38-49. doi: 10.1017/S0376892997000088

Garza-López, M., Ortega-Rodríguez, J. M., Zamudio-Sánchez, F. J., López-Toledo, J. F., Domínguez-Álvarez, F. A., \& SáenzRomero, C. (2016). Calakmul como refugio de Swietenia macrophylla King ante el cambio climático. Botanical Sciences, 94(1), 43-50. doi: 10.17129/botsci.500

Garza-López, M., Ortega-Rodríguez, J. M., Zamudio-Sánchez, F. J., López-Toledo, J. F., Domínguez-Álvarez, F. A., \& SáenzRomero, C. (2018). Modificación del hábitat para Lysiloma latisiliquum (1.) benth (Tzalam) por el cambio climático. Revista Fitotecnia Mexicana, 41(2), 127-135.
Gamfelt, L., Snäll, T., Bagchi, R., Jonsson, M., Gustafsson, L., Kjellander, P., Ruiz-Jaen, M. C., Fröberg, M., Stendahl, J., Philipson, C. D., \& Mikusiński, G. (2013). Higher levels of multiple ecosystem services are found in forests with more tree species. Nature Communications, 4(1340), 13-40. doi: $10.1038 /$ ncomms 2328

Gómez-Mendoza, L., Galicia, L., Aguilar-Santelises, R. (2008). Sensibilidad de grupos funcionales al cambio climático en la Sierra Norte de Oaxaca, México. Investigaciones Geográficas, 67(1), 76-100.

González de Andrés, E., Traba-Díaz, J., Seoane-Pinilla, J., \& MoralesPrieto, M. B. (2014). Aplicaciones de SIG y Teledetección en Ecología. Guion de prácticas de la asignatura, Universidad Autónoma de Madrid, Madrid, España.

González-Espinosa, M., Meave, J. A., Ramírez-Marcial, N., ToledoAceves, T., Lorea-Hernández, F. G., \& Ibarra-Manríquez, G. (2012). Los bosques de niebla de México: conservación y restauración de su componente arbóreo. Revista Ecosistemas, 21(12), 36-52.

Granados-Sánchez, D., López-Ríos, G. F., \& Hernández-García, M. A. (2007). Ecología y silvicultura en bosques templados. Revista Chapingo Serie Ciencias Forestales y del Ambiente, 13(1), 67-83.

Gutiérrez, E., \& Trejo, I. (2014). Efecto del cambio climático en la distribución potencial de cinco especies arbóreas de bosque templado en México. Revista mexicana de biodiversidad, 85(1), 179188. doi: $10.7550 / \mathrm{rmb} .37737$

Hijmans, R. J., Cameron, E. S., Parra, L. J., Jones, G. P., \& Jarvis, A. (2005). Very high-resolution interpolated climate surfaces for global land areas. International Journal of Climatology, 25(15), 19651978. doi: 10.1002/joc. 1276

Instituto Nacional de Estadística y Geografía [Inegi]. (2014). Encuesta Nacional de la Dinámica Demográfica: Porcentaje de población total en ámbito de localidad rural. Recuperado de https://www.inegi.org.mx/app/indicadores/?ind=6200112341 \&tm=7\#divFV62001123416200112341\#D6200112341

Instituto Nacional de Estadística y Geografía [Inegi]. (2016). Uso del suelo y vegetación, escala 1:250000, serie VI. Recuperado de http://www.conabio.gob.mx/informacion/metadata/gis/usv25 0s6gw.xml?_httpcache=yes\&_xsl=/db/metadata/xsl/fgdc_html .$x s l \& \_$indent $=$no.

Intergovernmental Panel on Climate Change [Ipcc]. (2007). Contribution of Working Groups I, II and III to the Fourth Assessment Report of the Intergovernmental Panel on Climate Change. Recuperado de https://www.ipcc.ch/assessment-report/ar4/

Lindner, M., Fitzgerald, J. B., Zimmermann, N. E., Reyer, C., Delzon, S., van der Maaten, E., \& Suckow, F. (2014). Climate change and 
European forests: what do we know, what are the uncertainties, and what are the implications for forest management?. Journal of environmental management, 146(1), 69-83. doi: 10.1016/j.jenvman.2014.07.030

Lloret, F. (2012). Vulnerabilidad y resiliencia de ecosistemas forestales frente a episodios extremos de sequía. Revista Ecosistemas, 21(3), 85-90. doi: 10.7818/ECOS.2012.21-3.11

Matías, L. (2012). Cambios en los límites de distribución de especies arbóreas como consecuencia de las variaciones climáticas. Revista Ecosistemas, 21(3), 91-96. doi: 10.7818/ECOS.2012.21-3.12

MaxEnt (2019). Maxent software for modeling species niches and distributions (Version 3.4.1). Recuperado de http://biodiversityinformatics.amnh.org/open_source/maxent/

McKenney, D. W., Pedlar, J. H., Lawrence, K., Campbell, K., \& Hutchinson, M. F. (2007). Potential impacts of climate change on the distribution of North American trees. BioScience, 57(11), 939948. doi: 10.1641/B571106

Millar, C. I., Stephenson, N. L., \& Stephens, S. L. (2007). Climate change and forests of the future: managing in the face of uncertainty. Ecological applications, 17(8), 2145-2151. doi: 10.1890/06-1715.1

Monterroso-Rivas, A., Gómez-Díaz, J., \& Tinoco-Rueda, J. (2013). Bosque mesófilo de montaña y escenarios de cambio climático: una evaluación en Hidalgo, México. Revista Chapingo Serie Ciencias Forestales y del Ambiente, 19(1), 29-43. doi: 10.5154/r.rchscfa.2012.03.029

Orellana, R., Espadas, C., Conde, C., \& Gay, C. (2012). Atlas: Escenarios de Cambio Climático en la Península de Yucatán. Recuperado de http://www.ccpy.gob.mx/agenda-regional/escenarios-cambioclimatico/atlas/

Phillips, S., Anderson, R., \& Schapire, R. (2006). Maximum entropy modeling of species geographic distributions. Ecological Modelling, 190(3-4), 231-259. doi: 10.1016/j.ecolmodel.2005.03.026

Pliscoff, P., \& Fuentes-Castillo, T. (2011). Modelación de la distribución de especies y ecosistemas en el tiempo y en el espacio: una revisión de las nuevas herramientas y enfoques disponibles. Revista de Geografia Norte Grande, 48(1), 61-79. doi: 10.4067/S071834022011000100005

Ramirez-Villegas, J., Jarvis, A., \& Läderach, P. (2013). Empirical approaches for assessing impacts of climate change on agriculture: The EcoCrop model and a case study with grain sorghum. Agricultural and Forest Meteorology, 170(1), 67-78. doi: 10.1016/j.agrformet.2011.09.005

Reece, J., \& Noss, R. (2014). Prioritizing species by conservation value and vulnerability: a new index applied to species threatened by sea-level rise and other risks in Florida. Natural Areas Journal, 34(1), 31-45. doi: 10.3375/043.034.0105

Seidl, R., Rammer, W., \& Spies, T. A. (2014). Disturbance legacies increase the resilience of forest ecosystem structure, composition, and functioning. Ecological Applications, 24(8), 2063-2077. doi: $10.1890 / 14-0255.1$

Van Zonneveld, M., Koskela, J., Vinceti, B., \& Jarvis, A. (2009a). Impact of climate change on the distribution of tropical pines in Southeast Asia. Unasylva, 231(232), 24-29.

Van Zonneveld, M., Jarvis, A., Dvorak, W., Lema, G., \& Leibing, C. (2009b). Climate change impact predictions on Pinus patula and Pinus tecunumanii populations in Mexico and Central America. Forest Ecology and Management, 257(7), 1566-1576. doi: 10.1016/j.foreco.2008.12.027

Wardle, A. D., \& Jonsson, M. (2014). Long-term resilience of above and belowground ecosystem components among contrasting ecosystems. Ecology, 95(7), 1836-1849. doi: 10.1890/13-1666.1

Warren, D. L., \& Seifert, S. N. (2011). Ecological niche modeling in Maxent: the importance of model complexity and the performance of model selection criteria. Ecological applications, 21(2), 335-342. doi: 10.1890/10-1171.1

WorldClim (2019). Global Climate Data:. Free climate data for ecological modeling and GIS. Recuperado de http://worldclim.org/version2

Zamora-Crescencio, P., García-Gil, G., Flores-Guido, J. S., \& Ortiz, J. J. (2008). Estructura y composición florística de la selva mediana subcaducifolia en el sur del estado de Yucatán, México. Polibotánica, 26(1), 39-66.

\section{Manuscrito recibido el 11 de febrero de 2020}

Aceptado el 21 de febrero de 2021

Publicado el 23 de diciembre de 2021

\section{Este documento se debe citar como:}

Santillán-Fernández, A. Escobar-Castillo, J., Ireta-Paredes, A. R., Espinosa-Grande, E., Bautista-Ortega, J., \& Chávez-Vergara, B. M. (2021). Relación entre la riqueza de especies arbóreas y el cambio climático en el sureste de México. Madera y Bosques, 27(3), e2732124. doi: 10.21829/myb.2021.2732124
Madera y Bosques por Instituto de Ecología, A.C. se distribuye bajo una Licencia Creative Commons Atribución-NoComercialCompartirlgual 4.0 Internacional. 